\title{
Research on English Translation Based on Functional Equivalence Theory and Genetic Algorithm
}

\author{
Yuan Liu 1 and Liangfeng Dong \\ Foreign Studies School of Xuzhou University of Technology, Xuzhou, Jiangsu 221000, China \\ Correspondence should be addressed to Yuan Liu; dlf@xzit.edu.cn
}

Received 11 October 2020; Revised 22 November 2020; Accepted 10 December 2020; Published 7 January 2021

Academic Editor: Hongju Cheng

Copyright (c) 2021 Yuan Liu and Liangfeng Dong. This is an open access article distributed under the Creative Commons Attribution License, which permits unrestricted use, distribution, and reproduction in any medium, provided the original work is properly cited.

\begin{abstract}
With the increasing development of our country and the world, the importance of English as an international language is selfevident. But we have difficulty in English translation, especially the vocabulary and translation of business English letters, not only because we have different living habits but also because we have different ways of speaking. Based on the research of functional equivalence theory and the calculation of a genetic algorithm, the vocabulary and translation of business English letters will be better improved. This can help us communicate better with each other and learn from the advanced Western experience in China. Through the study of the algorithm, the computational advantages of the algorithm are proved. The study of this English translation model will further improve the progress and promotion of existing translation technology.
\end{abstract}

\section{Introduction}

With the steady implementation of General Secretary Xi Jinping's strategy, China has become increasingly close to the international exchange [1,2]. English as the most widely circulated language, its practical significance is self-evident. However, because of the habits of our society and the different ways of life, we have caused a lot of communication obstacles, which seriously affect the communication between our country and the Western developed countries and hinder the cultural exchange and economic intercourse between our country and the countries of the world [3,4]. Not only that, the inaccurate translation may even cause some unpleasantness and contradictions. By using the theory of functional equivalence and the mutual application of genetic algorithms, this can help us to carry out empirical research on business English letter vocabulary and translation [5]. By combining functional equivalence theory with genetic algorithms, the credibility of our research is also increased. In this paper, the calculation of a genetic algorithm is studied [6,7]. First, from the calculation of the definition of genetic algorithm calculation and calculation steps to start research, the genetic algorithm research process and calculation method are understood; then, the genetic algorithm calculation for- mula and calculation steps are carried out in a comprehensive understanding of the calculation $[8,9]$.

Then, we build the mathematical model and compute the computer model according to the mathematical formula we calculate and optimize. At the end of the paper, the genetic algorithm is tested and calculated. The study of a genetic algorithm has increased the depth of our research and reduced the work pressure of our English translation work [10]. Based on the research of functional equivalence theory and the calculation of the genetic algorithm, the vocabulary and translation of business English letters will be better improved. This can help us communicate better with each other and learn from the advanced Western experience in China. Through the study of the algorithm, the computational advantages of the algorithm are proved. The study of this English translation model will further improve the progress and promotion of existing translation technology.

\section{Related Work}

Heredity is a kind of new computer algorithm that American scholar simplifies simulation according to the genetic system of biological in nature $[11,12]$. This new computer algorithm, which we study in the genetic system of biological 
organisms in nature, helps us to compute the various aspects according to the genetic laws of biology. Genetic algorithm is unanimously praised for its unique information processing mode. And we know that the way in which biological genetic systems are transmitted and the information processing patterns are excellent and accurate [13].

The study of the genetic model helps us to deal with some optimal values and the calculation of the optimal solution. Genetic algorithm has been widely referenced in various fields in the world by virtue of its powerful ability of optimal solution calculation. In China, in the '90s, the study of a genetic algorithm was introduced $[14,15]$. More than 20 years of computational learning has made our domestic research at the forefront of the world. Through continuous research, domestic scholars have developed several genetic algorithms suitable for our domestic use of the calculation version. In this paper, the calculation of a genetic algorithm is based on the domestic research of a genetic algorithm for purposeful optimization $[16,17]$. At present, the computational version of a genetic algorithm developed by domestic scholars has been gradually accepted by the whole world. Domestic research plays a pivotal role in the world.

\section{Methodology}

3.1. Application Steps of a Genetic Algorithm. The genetic algorithm used in this paper is not a classical genetic algorithm, and the genetic algorithm based on a traditional genetic algorithm is obtained [18]. But before we study the genetic algorithms used in this paper, traditional genetic algorithms need to be studied and analysed. Only after we have understood the computational process of traditional genetic algorithms can we optimize the study. The traditional computational process of a genetic algorithm is based on the genetic calculation of biological individuals. The genetic mutation process of genetic operators is also very simple, only by crossover, selection, and mutation three ways [19]. But for the common problems, the traditional algorithm can be solved completely. The traditional genetic algorithm mainly uses the genetic operator to compute the problem of our computation and can simplify the process of many very complicated computational problems. Then, the traditional computational process of a genetic algorithm will be studied by us [20].

For different problems, the computational process of a genetic algorithm has a certain difference. But the main computational process is the same, and we mainly analyse the common computational steps in our calculations. Usually, in the calculation, the genetic algorithm is calculated as follows: first, before the calculation, the decision variables in the calculation process need to be set by us. The decision variable determines whether the genetic operator in our calculations can be inherited. This is one of the key issues in our calculations $[21,22]$. The setting of decision variables directly affects the accuracy of our calculation results. If the decision variable is set incorrectly, then the calculation will not be the result we need, whether it is correct or not. The genetic variation of chromosomes is like the process shown in Figure 1.

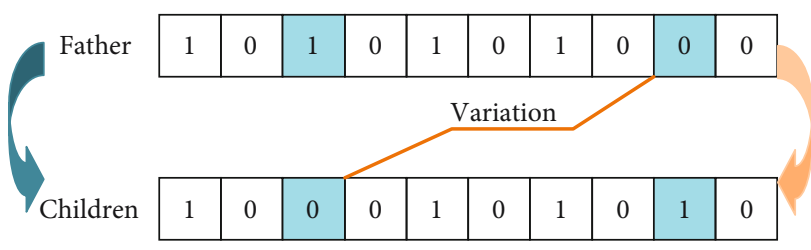

FIgURE 1: Chromosome genetic variation process.

The role of decision variables is a criterion of judgment. The data that is correct and we need will be intercepted, and the data that we do not need or incorrect will be deleted. Then, we can analyse the problem of solving a genetic algorithm. This part is the basis of our calculation, and the analysis of the problem of a genetic algorithm is a sort of our calculation purpose [23]. A clear calculation process and a calculated target will make our results more accurate. The problem of solving the genetic algorithm is the core of our calculation, and our calculation process revolves around the solution problem of our calculation. The process is to make our calculations clear by our research. Then, a study of individual forms of expression can be conducted. The individual representations of our calculations are determined [24].

Then, based on what we have studied above, the mathematical model can be built. For the establishment of the mathematical model, it is mainly to study the target illusion and the calculation of various formulas. The objective function is based on the analysis of the decision variables mentioned above. Our computational process has not really been completed after the mathematical model has been established $[25,26]$. We also need to build a computer computing model, because we know that genetic algorithms are computed by computer models. For the establishment of a computer computing model, we should mainly pay attention to the chromosome encoding and decoding process. Chromosomes are the carriers of our computational process, and the various computational analyses of genetic information in our calculations are based on chromosomes [27]. The coding process of chromosomes requires strict attention. Chromosome encoding should reflect the specific form and location of our genetic information. In addition, we need to match the decoding process. The computer calculates the result in the code form so that the computation result cannot be understood by the human. So, the reasonable decoding process needs to be developed according to the coding process, and the calculation result of the whole algorithm can be exported [28]. For the traditional genetic algorithm of the calculation process, it is shown in the form of Figure 2.

After the above content is set up, the evaluation method of the fitness needs to be set up. And then there is the genetic design. The genetic operator, which carries our computational content, is one of the key parts of our entire computational process $[29,30]$. The smallest unit of operation, such as crossover mutation, is the genetic operator, which is also the smallest unit of calculation in our calculations. So, the calculation process and design process of the whole genetic algorithm are studied. 


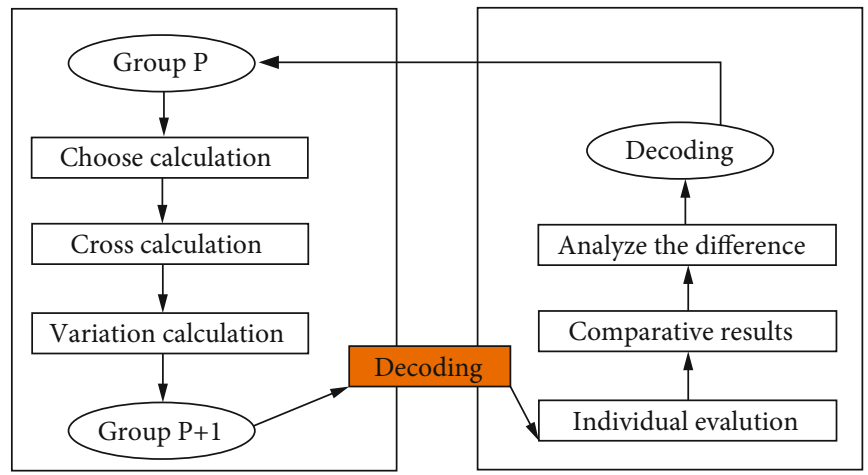

FIgURE 2: Traditional genetic algorithm calculation process.

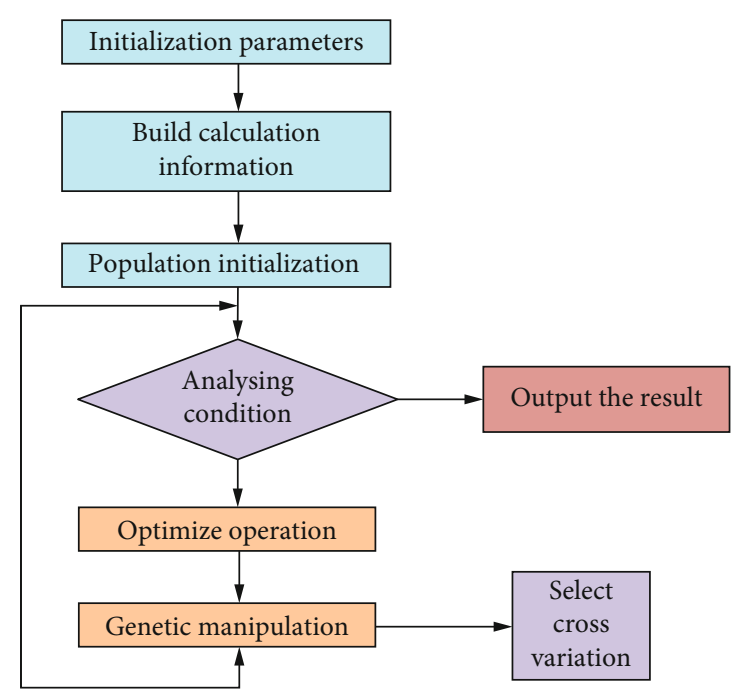

FIGURE 3: Mathematical model of genetic algorithms.

3.2. Mathematical Formula and Mathematical Model of a Genetic Algorithm. The calculation steps and design process of the genetic algorithm are studied, and the mathematical formula and mathematical model of the genetic algorithm are designed. The computational study of the mathematical model is mainly on the calculation of the fitness [31]. Biological heredity in nature is based on the rules of survival of the fittest. Highly adaptable individuals are more likely to inherit. The individual fitness is determined by the genetic operator, which is carried on the chromosome so that an individual is depicted step-by-step [32]. Next, the computational research and optimization process of the genetic algorithm are analysed.

First, genetic codes and new populations are established. A new population of chromosomes consisting of $N$ is randomly determined:

$$
\operatorname{pop}_{i}(t), \quad i=1,2,3, \cdots, N
$$

To calculate fitness, the fitness function is expressed as

$$
f_{i}=f \text { int ness }\left(\operatorname{pop}_{i}(t)\right)
$$

The probability of the fitness is

$$
P_{\mathrm{i}}=\frac{f_{i}}{\sum_{i=1}^{N} f_{i}} .
$$

The range of changes for each parameter is determined and is represented by a binary number. For example, if the variation range of the parameter a is $\left[a_{\min }, a_{\max }\right]$, it is represented by a binary $b$ of $m$ bit:

$$
a=a_{\min }+\frac{b}{2^{m}-1}\left(a_{\max }, a_{\min }\right) .
$$

At this point, the parameter range should cover all the optimization space. The determination of word length $m$ should be as small as possible in order to minimize the complexity of the genetic algorithm. The binary number that represents the parameter is threaded to form a long binary string, which is the object of the genetic algorithm [33].

There are usually two ways to produce an initial population:

(1) A completely random method can be used to produce a case without any prior knowledge

(2) For some prior knowledge, it is necessary to select samples randomly after satisfying the requirement of prior knowledge

The size of the fitness determines the probability that the individual is inherited into the next generation. The adaptability must be nonnegative, in order to satisfy this condition; the basic genetic algorithm uses the following method to transform [34].

$$
\begin{aligned}
& \operatorname{Fit}(f(x))=\frac{1}{1+C+f(x)}, \quad C \geq 0, C+f(x) \geq 0, \\
& \operatorname{Fit}(f(x))=\frac{1}{1+C-f(x)}, \quad C \geq 0, C+f(x) \geq 0 .
\end{aligned}
$$

The adaptability ratio method (fitness proportional model), also known as the betting wheel method, determines the selected probability of the individual according to the proportion of the whole population fitness [35]. If the 
TABLE 1: Optimization genetic algorithm and the advantages and disadvantages of the traditional genetic algorithm, a comparison table.

\begin{tabular}{lcc}
\hline Algorithm class & Disadvantages & Advantages \\
\hline \multirow{2}{*}{ Optimized genetic algorithm } & The calculation rules are cumbersome & Calculate a small amount \\
& Set up more trouble & High accuracy \\
Traditional genetic algorithm & Accuracy at the end & Eliminate, do not repeat the calculation \\
& Calculate for a long time & Longer use \\
\hline
\end{tabular}

TABLE 2: Function test parameter setting table.

\begin{tabular}{lcccc}
\hline $\begin{array}{l}\text { Function } \\
\text { parameter }\end{array}$ & $\begin{array}{c}\text { Population } \\
\text { size }\end{array}$ & $\begin{array}{c}\text { Binary } \\
\text { code } \\
\text { length }\end{array}$ & $\begin{array}{c}\text { The } \\
\text { maximum } \\
\text { genetic } \\
\text { algebra }\end{array}$ & $\begin{array}{c}\text { Variation in the } \\
\text { operation of the } \\
\text { parameters }\end{array}$ \\
\hline$F(1)$ & 100 & 20 & 200 & $K=0.1$ \\
$F(2)$ & 100 & 20 & 200 & $K=0.1$ \\
$F(3)$ & 100 & 20 & 500 & $K=0.05$ \\
$F(4)$ & 100 & 20 & 500 & $K=0.05$ \\
$F(5)$ & 200 & 20 & 500 & $K=0.05$ \\
\hline
\end{tabular}

population number is $n$ and the fitness of the individual $i$ is $f(i)$, then the probability of the individual i selection is $\mathrm{p}$. The total probability of the individual is $Q_{t}$, which determines which individual participates in mating through probability calculation and allocation. The probability of the individual i being selected is $\mathrm{p}$. The calculation formula of the individual total probability is as follows:

$$
\begin{gathered}
P_{i}=\frac{f_{i}}{\sum_{i=1}^{N} f_{i}}, \\
Q_{\mathrm{t}}=\sum_{j=1}^{i} p_{j}, \quad j=1,2,3, \cdots, i .
\end{gathered}
$$

In the above calculation process, not only the traditional genetic algorithm of the computational process has been completed but also the genetic algorithm has been reasonably optimized. The mathematical model of the genetic algorithm is analysed in Figure 3.

Through our genetic algorithm optimization, it is more suitable for the calculation of the use of this article. The accuracy of the whole calculation process is increased. The advantages of our optimization are shown in Table 1.

\section{Result Analysis and Discussion}

In the computational research above, the computational procedure of the genetic algorithm and the calculation formula and the computer model are completed. However, the algorithm needs to be tested properly before it is put into use. Because each algorithm has a certain flaw, the test of the algorithm is to find the relatively weak link to continue optimization research. Then, we began to design the experimental process, the test process. Five groups of experiments were established. Five groups of experiments each group except our unique variable, the other test environment is the same. We mainly study the accuracy of calculation and the change of population mean value and adaptability. The parameters we tested are designed as shown in Table 2.

The data in the table above is entered into the computer calculation model. Then, we began to design the experimental process, the test process. Five groups of experiments were established. The five groups of experiments were calculated and shaped five times and then averaged. In the computational research above, the computational procedure of the genetic algorithm and the calculation formula and the computer model are completed. After decoding the results, the results are shown in Table 3.

For the analysis of the experimental data in Table 3, we can know that our optimized genetic algorithm can perfectly eliminate the problem of local minima in traditional genetic algorithm calculation. The calculated results in the table are consistent with the results we have already known. It can be found that the computational accuracy of the optimized genetic algorithm can be higher than that of the traditional genetic algorithm by $23 \%$. This is a great advantage, the traditional genetic algorithm $74 \%$ to improve the accuracy of the calculation with $97 \%$, which basically meets our testing requirements. Research on the Identification of English Translation Efficiency is shown in Figure 4.

We specify that the calculation accuracy of more than $95 \%$ is called to be qualified. The computational accuracy of the optimized algorithm is fully qualified. In this paper, we also find a point in the calculation process, that is, we optimize the genetic algorithm after the calculation time is only one-third of the traditional algorithm. This greatly shortens the time we calculate so that we can compute more data within the same time. In addition, the minimum fitness of 0 to 80 computational individuals has also been tested. The minimum degree of fitness is related to the number of iterations we calculate. The greater the degree of adaptability, the shorter the time that the result of our calculations will appear. It means that the shorter the number of iterations, the shorter the computation time. The change of the minimum fitness to the increase in the number of individuals is shown in Figure 5.

In Figure 4, we can see that as the computational individual increases, the degree of adaptability is reduced and concentrated. The calculation result is unique, but the increase of the calculated item results in the decrease of the fitness average. We can see that the degree of adaptability is constantly concentrated as the number of items increases. This shows that the calculated time does not increase significantly 
TABLE 3: Contrast experimental calculation result comparison table.

\begin{tabular}{lcccc}
\hline \multirow{2}{*}{ Function } & \multicolumn{2}{c}{ Traditional genetic algorithm } & \multicolumn{2}{c}{ Optimized genetic algorithm } \\
The optimal value & The optimal value \\
\hline$F(1)$ & $(-0.073314,1.521994)$ & 8.105630 & $(1.564704,0.01176)$ & 8.09791 \\
$F(2)$ & $(-0.000000,0.000000)$ & 1.000000 & $(-0.0022775,-0.0021200)$ & 0.999990 \\
$F(3)$ & $(0.976540,1.000000)$ & 0.000000 & $(1.002351,1.000168)$ & 0.018176 \\
$F(4)$ & $(0.087977,-0.713587)$ & -1.031606 & $(0.0998189,-0.7107720)$ & -1.031193 \\
$F(5)$ & $(-0.073314,1.521994)$ & 7.105630 & $(1.564704,0.01176)$ & 7.09791 \\
\hline
\end{tabular}

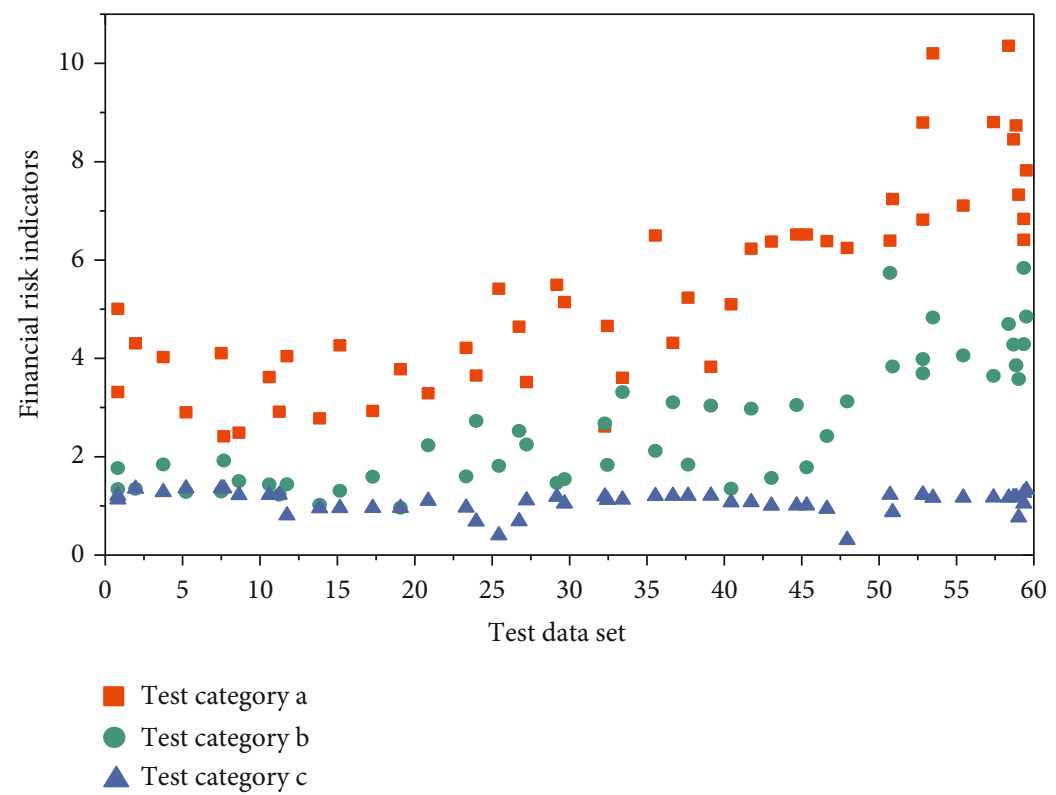

FIgURE 4: Research on the Identification of English Translation Efficiency.

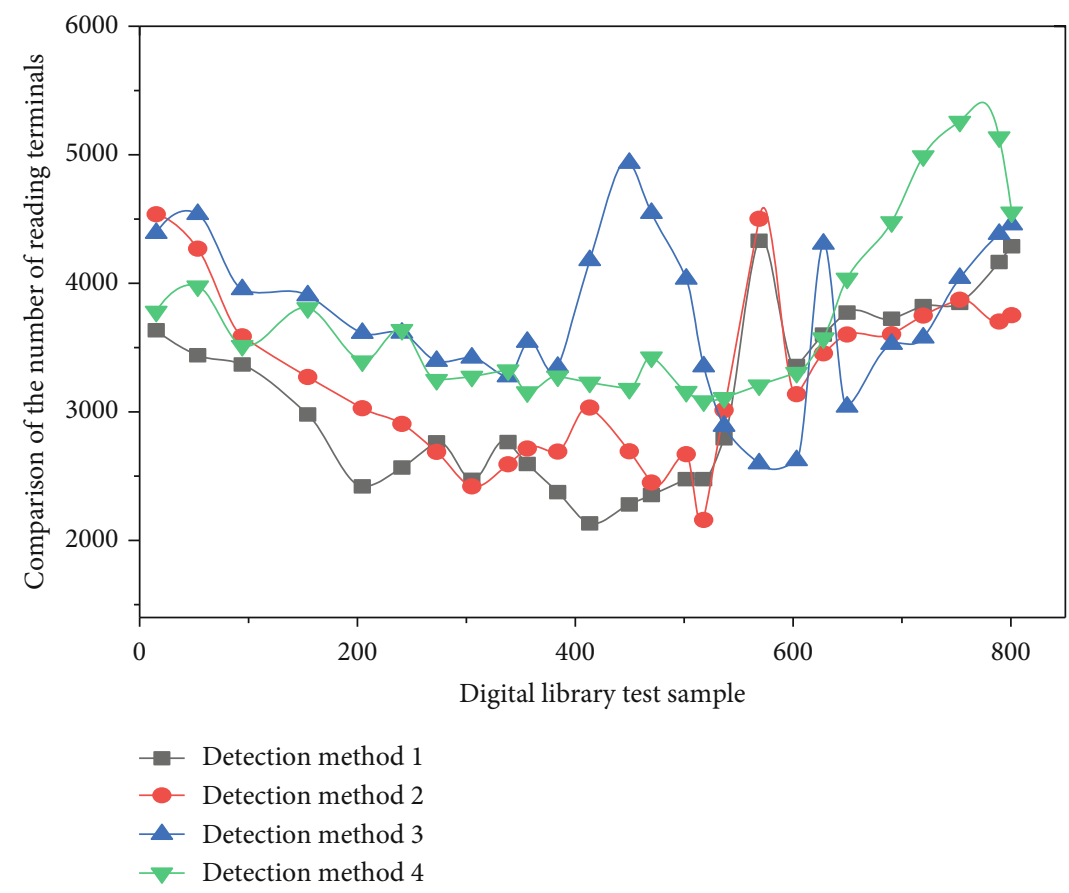

FIGURE 5: Changes in the fitness of the smallest number of individuals against the increase in the number of cases. 


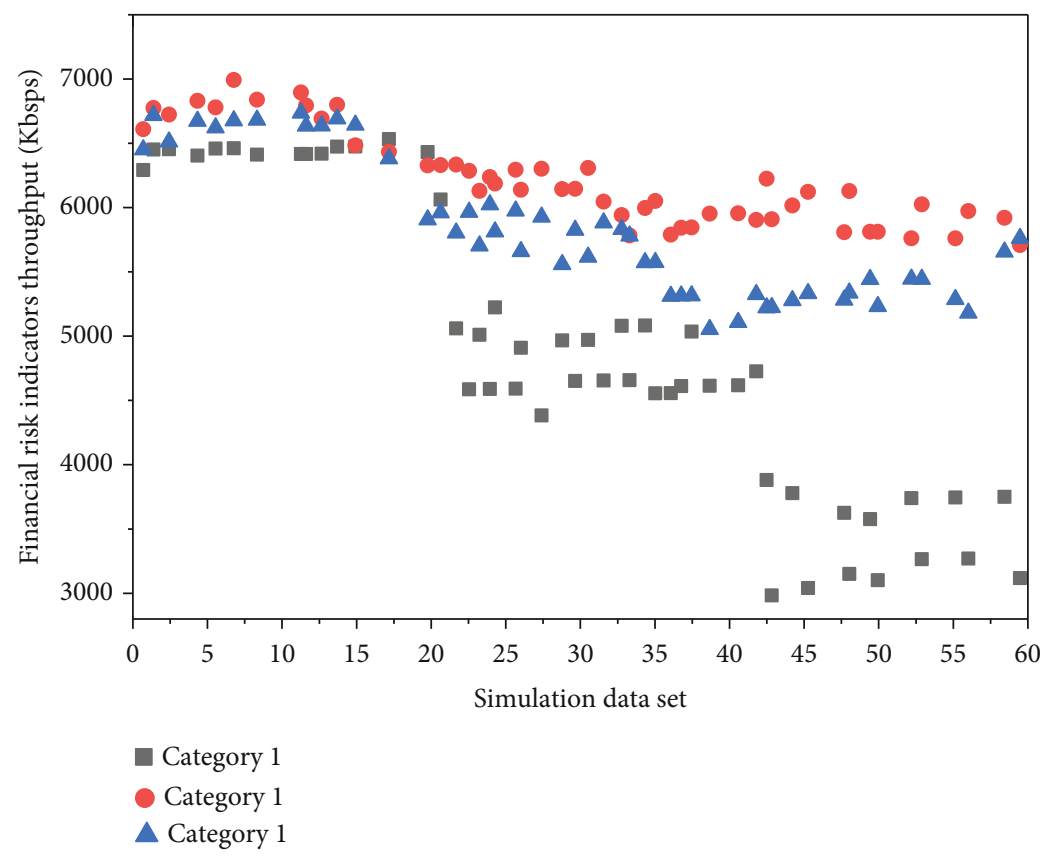

FIgURE 6: Variation of the population mean value as the number of iterations increases.

because of the increased number of calculated items. This greatly shortens the time we calculate so that we can compute more data within the same time. In addition, the minimum fitness of 0 to 80 computational individuals has also been tested. The minimum degree of fitness is related to the number of iterations we calculate. It is proved that our optimized algorithm can deal with many individual computations. In addition, the change of mean size as the number of iterations increases is also studied. The number of iterations is set to 100. The changes of the population mean change with the increase of the number of iterations were observed. The changes are shown in Figure 6.

From the graph, we can see that with the increase of the number of iterations, the change of the group mean is decreasing with the increase of the number of iterations. But the population mean is more and more concentrated. Our results are output in the form of an individual, so the image is a single point, not a curve. The mean of the group is more and more concentrated, which shows that we can solve the difficult problem in our calculation, and the optimized algorithm is still able to calculate the result. And we can figure out how many sets of results we need.

\section{Conclusions}

Today, the communication between the state and the state is becoming more and closer. In addition, English as the universal language internationally, its importance is selfevident. However, because of social and various reasons, it is difficult for us to translate English documents accurately. Therefore, the theory of functional equivalence was studied and combined with a genetic algorithm to help us to prove the study of translation. Through our test of the algorithm, we find that the average computational accuracy of our optimized genetic algorithm can be higher than that of the tradi- tional genetic algorithm 23\%. The calculation accuracy of the traditional genetic algorithm $74 \%$ is increased by $97 \%$. And the calculation time of the whole calculation is shortened to one-third of the original algorithm. And through our optimization, the problem of iterative times of the genetic algorithm is reduced greatly, and the problem of local minima is eliminated. In addition, our optimized algorithm can still calculate the result for the difficult problem in our calculation. This is a great advantage of our optimized genetic algorithm.

\section{Data Availability}

All the data are authentic and reliable, and the sources are available from official sources.

\section{Conflicts of Interest}

The authors declare no conflict of interest.

\section{Acknowledgments}

This paper is supported by Jiangsu Provincial Higher Educational Philosophy and Social Research Programme (2019SJA0979).

\section{References}

[1] Z. Yan Kai, F. Sze Ming, J. Nyan Ping, C. Po Yu, and L. Albert, "Experimentally-implemented genetic algorithm (Exp-GA): toward fully optimal photovoltaics," Optics Express, vol. 23, pp. A1324-A1333, 2018.

[2] E. R. Thomas, "Sociophonetic trends in studies of Southern U.S. English," Journal of the Acoustical Society of America, vol. 147, no. 1, pp. 529-540, 2020. 
[3] A. Stevanovic, P. T. Martin, and J. Stevanovic, "VisSim-based genetic algorithm optimization of signal timings," Transportation Research Record, vol. 2035, pp. 59-68, 2018.

[4] Z. Song, "English speech recognition based on deep learning with multiple features," Computing, vol. 102, no. 3, pp. 663682, 2020

[5] D. V. René and H. Fred, "English translation of the Dutch blood transfusion guideline 2011," Clinical Chemistry, vol. 58, pp. 1266-1267, 2020.

[6] P. Li, G. C. Constantinescu, N.-T. A. Nguyen, and C. C. Jeffery, "Trends in reporting of swallowing outcomes in oropharyngeal cancer studies: a systematic review," Dysphagia, vol. 35, pp. 18-23, 2019.

[7] J. N. E. Ölmestig, I. R. Marlet, A. H. Hainsworth, and C. Kruuse, "Phosphodiesterase 5 inhibition as a therapeutic target for ischemic stroke: a systematic review of preclinical studies," Cellular Signalling, vol. 38, pp. 39-48, 2017.

[8] P. Olen, "A forgotten strand of reception history: understanding pure semantics," Synthese, vol. 194, pp. 121-141, 2017.

[9] I. Mitxelena and M. Piris, "Analytic gradients for spin multiplets in natural orbital functional theory," Journal of Chemical Physics, vol. 153, no. 4, article 044101, 2020.

[10] L. Michaeli and A. Bahabad, "Genetic algorithm driven spectral shaping of supercontinuum radiation in a photonic crystal fiber," Journal of Optics, vol. 20, no. 5, article 055501, 2018.

[11] Z. Liu, "A study on English translation of tourism publicity in coastal cities from the perspective of cross-cultural communication," Journal of Coastal Research, vol. 115, no. sp1, p. 87, 2020.

[12] N. Lazarovich and A. Levit, "Edge Kempe equivalence of regular graph covers," Journal of Graph Theory, vol. 93, pp. 553$559,2019$.

[13] P. Klein, A. Friedman, M. Q. Hameed et al., "Repurposed molecules for antiepileptogenesis: missing an opportunity to prevent epilepsy?," Epilepsia, vol. 61, pp. 359-386, 2020.

[14] W. H. Jost, A. Rizos, P. Odin, M. Löhle, and A. Storch, "King's Parkinson's disease pain scale: intercultural adaptation in the German language," Nervenarzt, vol. 89, no. 2, pp. 178-183, 2018.

[15] S. Jinyoung, G. Aejin, K. Hyeonyoung et al., "Validation study for the Korean version of fear of cancer recurrence inventory," Journal of Korean Medical Science, vol. 32, no. 11, pp. 17921799, 2017.

[16] J. H. Jensen, “A graph-based genetic algorithm and generative model/Monte Carlo tree search for the exploration of chemical space," Chemical Science, vol. 10, no. 12, pp. 3567-3572, 2019.

[17] H. J. Jensen, "A graph-based genetic algorithm and generative model/Monte Carlo tree search for the exploration of chemical space," Chemical Science, vol. 10, pp. 3567-3572, 2019.

[18] J.-F. Danel and L. Kazandjian, "A simple scaling law for the equation of state and the radial distribution functions calculated by density-functional theory molecular dynamics," Physics of Plasmas, vol. 25, no. 6, article 060702, 2018.

[19] K. Indrek and F. Mike, "Omics approaches for subcellular translation studies," Molecular Omics, vol. 14, pp. 380-388, 2018.

[20] H. Hong, M. Panahi, A. Shirzadi et al., "Flood susceptibility assessment in Hengfeng area coupling adaptive neuro-fuzzy inference system with genetic algorithm and differential evolution," Science of the Total Environment, vol. 621, pp. 1124$1141,2018$.
[21] P. Gooding, B. Mcsherry, and C. Roper, "Preventing and reducing "coercion" in mental health services: an international scoping review of English-language studies," Acta Psychiatrica Scandinavica, vol. 142, no. 1, pp. 27-39, 2020.

[22] D. W. Gong, J. Sun, and Z. Miao, "A set-based genetic algorithm for interval many-objective optimization problems," IEEE Transactions on Evolutionary Computation, vol. 22, no. 1, pp. 47-60, 2018.

[23] M. Giassi and M. Goteman, "Layout design of wave energy parks by a genetic algorithm," Ocean Engineering, vol. 154, pp. 252-261, 2018.

[24] C. Figdor, "On the proper domain of psychological predicates," Synthese, vol. 194, no. 11, pp. 4289-4310, 2017.

[25] M. V. J. Felipe, Y. A. Kinkhabwala, S. Jeffrey, C. Itai, and T. A. Arias, "Density-functional fluctuation theory of crowds," Nature Communications, vol. 9, no. 1, p. 3538, 2018.

[26] A. Ebrahim, T. S. H. Martin, P. J. Mumby, A. D. Olds, and I. R. Tibbetts, "Differences in diet and foraging behaviour of commercially important rabbitfish species on coral reefs in the Indian Ocean," Coral Reefs, vol. 39, pp. 977-988, 2020.

[27] B. Doerr and C. Doerr, "Optimal static and self-adjusting parameter choices for the $(1+(\lambda, \lambda))$ genetic algorithm," Algorithmica, vol. 80, no. 5, pp. 1658-1709, 2018.

[28] K. N. Dew, A. M. Turner, Y. K. Choi, B. Alyssa, and K. Katrin, "Development of machine translation technology for assisting health communication: a systematic review," Journal of Biomedical Informatics, vol. 85, pp. 56-67, 2018.

[29] S. E. Detiger, A. F. Karim, R. M. Verdijk, P. M. V. Hagen, and D. Paridaens, "The treatment outcomes in IgG4-related orbital disease: a systematic review of the literature," Acta Ophthalmologica, vol. 97, no. 5, pp. 451-459, 2019.

[30] D. Deng and N. Xue, "Translation divergences in ChineseEnglish machine translation: an empirical investigation," Computational Linguistics, vol. 43, pp. 521-565, 2017.

[31] P. Das, A. Kuznetsova, M. O. Zhu, and R. Milanaik, "Dangers of machine translation: the need for professionally translated anticipatory guidance resources for limited English proficiency caregivers," Clinical Pediatrics, vol. 58, no. 2, pp. 247-249, 2018.

[32] C. Contaldi, F. Vafaee, and P. C. Nelson, "Bayesian network hybrid learning using an elite-guided genetic algorithm," Artificial Intelligence Review, vol. 52, pp. 245-272, 2018.

[33] Z. Chen, G. Laura, and T. Donald, "Multiconfiguration pairdensity functional theory for iron porphyrin with CAS, RAS, and DMRG active spaces," Journal of Physical Chemistry A, vol. 123, pp. 3389-3394, 2019.

[34] J. Carson, D. Cenzer, and J. B. Remmel, "Effective categoricity of automatic equivalence and nested equivalence structures," Theory of Computing Systems, vol. 64, pp. 1110-1139, 2020.

[35] H. Aziza and S. Krichen, "Bi-objective decision support system for task-scheduling based on genetic algorithm in cloud computing," Computing, vol. 100, no. 2, pp. 65-91, 2018. 